\title{
A NOTE ON THE PAPER OF Y. NAITO
}

\author{
PIOTR BILER \\ Instytut Matematyczny, Uniwersytet Wrocławski \\ Pl. Grunwaldzki 2/4, 50-384 Wrocław, Poland \\ E-mail: Piotr.Biler@math.uni.wroc.pl
}

\begin{abstract}
This note contains some remarks on the paper of Y. Naito concerning the parabolic system of chemotaxis and published in this volume.
\end{abstract}

1. Introduction. The aim of this note is to comment on some results of Yùki Naito in [6] and (with coauthors) in [7]. The author proves that if the total mass $0 \leq \widehat{M}=\int_{\mathbb{R}^{2}} u$ is small enough, then solutions of the parabolic system of chemotaxis

$$
\begin{aligned}
\frac{\partial u}{\partial t} & =\nabla \cdot(\nabla u-u \nabla v), \quad x \in \mathbb{R}^{2}, t>0, \\
\tau \frac{\partial v}{\partial t} & =\Delta v+u, \quad x \in \mathbb{R}^{2}, t>0,
\end{aligned}
$$

with $\tau=1$, cf. (1.1) in [6], behave asymptotically like self-similar solutions of that system. Here, we consider a more general system with the parameter $\tau>0$ as in [7].

The case of the parabolic-elliptic system with $\tau=0$ has been considered in [4] and [3]. The authors of [4] proved asymptotically self-similar behavior of solutions of (1)-(2) using entropy methods. We studied in [3] radially symmetric solutions of that system and proved that for each $0 \leq \widehat{M}<8 \pi$ (so in the whole range of the existence of self-similar solutions), the asymptotics of those solutions is determined by the unique self-similar solution $m_{\widehat{M}}$ corresponding to the mass $\widehat{M}$ :

$$
\lim _{t \rightarrow \infty}\left\|M(\cdot, t)-m_{\widehat{M}}(t)\right\|_{L^{\infty}}=0 .
$$

Here the cumulated density $M=M(s, t)$ is defined by $M(s, t)=\int_{B(0, \sqrt{s})} u(x, t) d x$, and satisfies the parabolic equation

$$
M_{t}=4 s M_{s s}+\frac{1}{\pi} M M_{s}
$$

2000 Mathematics Subject Classification: 35Q, 35K60, 35B40, 82C21.

Key words and phrases: self-similar solutions, parabolic chemotaxis system.

The paper is in final form and no version of it will be published elsewhere. 
supplemented with the boundary and initial conditions

$$
\begin{gathered}
M(0, t)=0, \quad M(\infty, t) \equiv \lim _{s \rightarrow \infty} M(s, t)=\widehat{M}, \\
M(s, 0)=M_{0}(s),
\end{gathered}
$$

the function $M_{0}$ being a nondecreasing continuous function on $(0, \infty)$. The self-similar solution $m=m(s / t)$ satisfies the equation

$$
4 m^{\prime \prime}(y)+m^{\prime}(y)+\frac{1}{\pi y} m(y) m^{\prime}(y)=0, \quad m(0)=0, \quad m(\infty)=\widehat{M},
$$

where $y=s / t, \quad '=d / d y$. It was proved in [1], [3] that given $0 \leq \widehat{M}<8 \pi$ self-similar solutions defined in (6) exist, have the finite derivative at $y=0: m^{\prime}(0)<\infty$, and they are unique: $m=m_{\widehat{M}}$. For $\widehat{M}>8 \pi$ there are no solutions of (6) nor even global in time solutions of $(3)-(5)$.

For the parabolic system (1)-(2) the determination of the optimal range of $\widehat{M}$ leading to the existence of self-similar solutions for a given $\tau$ and their uniqueness with a given $\widehat{M}$ seem to be an open problem. They are determined by the equation

$$
\begin{array}{r}
\Phi^{\prime \prime}+\frac{1}{4} \Phi^{\prime}+\frac{1}{2 y} \Phi^{\prime} e^{-\tau y / 4}\left(\int_{0}^{y} e^{\tau z / 4} \Phi^{\prime}(z) d z\right)=0, \\
\Phi(0)=0, \quad \Phi(\infty)=\frac{\widehat{M}}{2 \pi} .
\end{array}
$$

The best published results in this direction are in [7], where preliminary estimates from [2] for $\tau=1$ are greatly improved. In particular, the authors of [7] proved that

- if $0<\tau \leq 1 / 2$, then solutions of (7) exist exactly for $\widehat{M} \in[0,8 \pi)$,

$\bullet \bullet$ if $\tau \in(1 / 2,1]$ then $\widehat{M}<4 \pi^{3} / 3$,

$\bullet \bullet \bullet$ if $\tau>1$ then $\widehat{M}<\tau^{2} 4 \pi^{3} / 3$.

The solutions of (7) are smooth as a consequence of an analysis of the self-similar solutions of the original system (1)-(2) without radial symmetry assumptions. In fact, each self-similar solution of (1)-(2) is radial, [7, Th. 3]. A different construction of selfsimilar solutions of (1)-(2) was in [2].

Here we prove in a direct way that $\Phi^{\prime}(0)<\infty$ for solutions of $(7)$; direct means here: not using the correspondence between the densities $(u, v)$ in $(1)-(2)$ and the cumulated densities $(\varphi, \psi)$ in $(8)$ below. Moreover, we simplify the arguments leading to $\bullet$ (Lemma 2.4 ), and improve $\bullet \bullet \bullet$ (Lemma 2.5).

The results in [6, Th. 3] are proved using perturbation arguments via the contraction mapping theorem. This permitted the author to obtain uniqueness of sufficiently small self-similar solutions. The problem of the uniqueness of self-similar solutions is mentioned in [6, Remark 1 (i)]. Such a property would permit one to prove a stronger version of Theorem 3 in [6], i.e. the asymptotics of any global in time solution $u$ is described by that of the unique self-similar solution corresponding to the same mass $\widehat{M}$ as that for $u$, see remarks after Proposition 2.6 below.

We believe that this property holds for (7) but this conjecture is far from being obvious since for some nonlinear parabolic equations there are multiple self-similar solutions, see 
e.g. [5]. Moreover, it was shown in [7, Th. 2] that self-similar solutions form a oneparameter family, and their $L^{1}$ norms tend to $8 \pi$ as the parameter goes to $\infty$. Thus, the uniqueness does not hold if there is a self-similar solution for $\widehat{M}>8 \pi$.

2. Properties of self-similar profiles. Proceeding similarly to [7] or [3] we obtain the system for the cumulated densities

$$
\varphi(s, t)=\frac{1}{2 \pi} \int_{B(0, \sqrt{s})} u(x, t) d x, \quad \psi(s, t)=\frac{1}{2 \pi} \int_{B(0, \sqrt{s})} v(x, t) d x,
$$

equivalent for sufficiently smooth solutions to the original system (1)-(2)

$$
\begin{aligned}
\varphi_{t} & =4 s \varphi_{s s}-2 \varphi_{s}\left(s \psi_{s s}\right), \\
\tau \psi_{t} & =4 s \psi_{s s}+\varphi,
\end{aligned}
$$

with the boundary conditions

$$
\varphi(0, t)=\psi(0, t)=0, \quad \varphi(\infty, t)=\frac{\widehat{M}}{2 \pi} .
$$

This is, of course, a counterpart of (3) for the parabolic-elliptic system of chemotaxis. A self-similar solution $(u, v)$ of (1)-(2) satisfying the scaling property $\lambda^{2} u\left(\lambda x, \lambda^{2} t\right) \equiv$ $u(x, t), v\left(\lambda x, \lambda^{2} t\right) \equiv v(x, t)$ for each $\lambda>0$ corresponds to the functions $\varphi(s, t)=\Phi(s / t)$ and $\psi(s, t)=t \Psi(s / t)$ solving the system

$$
\begin{aligned}
& \Phi^{\prime \prime}+\frac{1}{4} \Phi^{\prime}-2 \Phi^{\prime} \Psi^{\prime \prime}=0 \\
& 4 y \Psi^{\prime \prime}+\tau y \Psi^{\prime}-\tau \Psi+\Phi=0
\end{aligned}
$$

where $y=s / t$. Here, the boundary conditions become

$$
\Phi(0)=\Psi(0)=0, \quad \Phi(\infty)=\frac{\widehat{M}}{2 \pi} .
$$

Putting $S(y)=\Phi(y)-\tau \Psi(y)+\tau y \Psi^{\prime}(y)=-4 y \Psi^{\prime \prime}(y)$ in (9)-(10) we obtain a simpler equation for $\Phi$, the counterpart of (6) for $m$ in the case $\tau=0$

$$
\begin{aligned}
& \Phi^{\prime \prime}+\frac{1}{4} \Phi^{\prime}+\frac{1}{2 y} \Phi^{\prime} S=0 \\
& S^{\prime}+\frac{\tau}{4} S=\Phi^{\prime}, \\
& \Phi(0)=0, \quad \Phi(\infty)=\frac{\widehat{M}}{2 \pi}, \quad S(0)=0 .
\end{aligned}
$$

To justify the boundary condition for $S$ observe that $\Phi^{\prime}(y), \Psi^{\prime}(y)>0$ for each $y>0$ leads to $\liminf _{y \rightarrow 0} S(y) \geq 0$, and thus, by $(12), S(y)>0$ for each $y>0$. Since by the definition (10) $S(y)=-4 y \Psi^{\prime \prime}(y), \Psi$ is concave, so that $0<\Psi^{\prime}(y) \leq \frac{\Psi(y)}{y}$, and $\lim _{y \rightarrow 0} y \Psi^{\prime}(y)=0$ follows. Similarly, the concavity of $\Phi$ and the relation

$$
\lim _{y \rightarrow 0} y \Phi^{\prime}(y)=0
$$

are obtained from (11) (a more precise argument repeats the lines of the proof of Lemma 4.1 in $[3])$. 
The above system (11)-(12) can be put either in the form of a first order differential equation for $\Phi^{\prime}$ (cf. (7)) with the integral term

$$
S(y)=e^{-\tau y / 4} \int_{0}^{y} e^{\tau z / 4} \Phi^{\prime}(z) d z,
$$

or a second order ordinary differential equation for $S$

$$
S^{\prime \prime}+\frac{\tau+1}{4} S^{\prime}+\frac{\tau}{16} S+\frac{1}{2 y}\left(S S^{\prime}+\frac{\tau}{4} S^{2}\right)=0 .
$$

LEMMA 2.1. The profile $\Phi$ satisfying (11) is increasing, concave, belongs to the space $\mathcal{C}^{\infty}((0, \infty)) \cap \mathcal{C}^{1}([0, \infty))$ and satisfies

$$
\frac{\widehat{M}}{2 \pi}\left(1-e^{-y / 4}\right) \leq \Phi(y) \leq \min \left(4 \Phi^{\prime}(0)\left(1-e^{-y / 4}\right), \frac{\widehat{M}}{2 \pi}\right)
$$

for every $y \in(0, \infty)$.

Proof. First note that $S(y)>0$ for each $y>0$. Otherwise for $y_{1}=\inf \{y>0: S(y)=0\}$ we would have $S^{\prime}\left(y_{1}\right) \leq 0$ contradicting (12). Recall that $\Phi^{\prime \prime}(y)<0$ because $\Phi^{\prime}(y)>0$ and $S(y)>0$. Since $\Phi^{\prime}-S^{\prime}=\frac{\tau}{4} S>0, S(y) \leq \Phi(y)$, the function $S$ is also strictly increasing and concave on a small interval $\left(0, y_{0}\right)$ (but not on the whole positive half-line $(0, \infty))$. Therefore,

$$
\frac{\Phi\left(y_{*}\right)}{y_{*}} \geq \frac{S\left(y_{*}\right)}{y_{*}} \geq \frac{S(y)}{y} \geq S^{\prime}(y)>0
$$

for all $0<y_{*}<y \leq y_{0}$. Now, (11) implies the inequality $4 \Phi^{\prime \prime}+\Phi^{\prime}+2 \Phi^{\prime} \frac{S\left(y_{*}\right)}{y_{*}} \geq 0$. After integration on $\left(y_{*}, y\right)$

$$
4 \Phi^{\prime}(y)+\Phi(y)\left(1+2 \frac{S\left(y_{*}\right)}{y_{*}}\right) \geq 4 \Phi^{\prime}\left(y_{*}\right)+\Phi\left(y_{*}\right)\left(1+2 \frac{S\left(y_{*}\right)}{y_{*}}\right) .
$$

Next, consider the function $w(y)=\Phi(y)-4 y \Phi^{\prime}(y), w(0)=0$ by (13). Since $w^{\prime}(y)=$ $\Phi^{\prime}(y)(-3+y+2 S(y))$, it is clear that $w^{\prime}(y)<0$ for all $y \in\left(0, y_{c}\right)$ with some $y_{c}>0$ and $w^{\prime}(y)>0$ for large $y$. Thus we have

$$
\frac{S\left(y_{*}\right)}{y_{*}} \leq \frac{\Phi\left(y_{*}\right)}{y_{*}} \leq 4 \Phi^{\prime}\left(y_{*}\right) \quad \text { for each } y_{*} \in\left(0, y_{c}\right) .
$$

As a consequence of (17) we obtain

$$
4 \Phi^{\prime}(y)+\Phi(y)\left(1+8 \Phi^{\prime}\left(y_{*}\right)\right) \geq 4 \Phi^{\prime}\left(y_{*}\right)
$$

or $4 \Phi^{\prime}(y)+\Phi(y) \geq 4 \Phi^{\prime}\left(y_{*}\right)(1-2 \Phi(y))$. Take $y>0$ small enough such that $\Phi(y)<\frac{1}{2}$. Since $\Phi^{\prime}(y)-S^{\prime}(y)=\frac{\tau}{4} S(y)=o(1)$ as $y \rightarrow 0$, passing to the limit $y_{*} \rightarrow 0$ in (18) we get $\lim _{y \rightarrow 0} \Phi^{\prime}(y)=\lim _{y \rightarrow 0} S^{\prime}(y) \in(0, \infty)$.

Evidently, it follows from (11) that $\Phi$ is a supersolution to the linear problem

$$
-4 \eta^{\prime \prime}-\eta^{\prime}=0, \quad \eta(0)=0, \quad \eta(\infty)=\frac{\widehat{M}}{2 \pi},
$$

and the comparison principle ensures that $\Phi(y) \geq \Phi(\infty)\left(1-e^{-y / 4}\right)$. On the other hand, the inequality $4 \Phi^{\prime \prime}+\Phi^{\prime} \leq 0$ implies

$$
\Phi^{\prime}(y) \leq \Phi^{\prime}(0) e^{-y / 4},
$$


so in particular $\Phi^{\prime}(y) \rightarrow 0$ as $y \rightarrow \infty$. Therefore, the inequality $\Phi(y) \leq 4 \Phi^{\prime}(0)\left(1-e^{-y / 4}\right)$ is satisfied.

A more precise asymptotic formula for $\Phi^{\prime}$ can be obtained from (11) written as

$$
\begin{aligned}
0 & =\frac{\Phi^{\prime \prime}(y)}{\Phi^{\prime}(y)}+\frac{1}{4}+\frac{S(y)}{2 y} \\
& =\frac{d}{d y}\left(\log \left(\Phi^{\prime}(y)\right)+\frac{y}{4}+\int_{1}^{y} \frac{S(z)}{2 z} d z\right) ;
\end{aligned}
$$

note that $\Phi^{\prime}(y)>0$ for $y>0$. Integrating this on $(1, y)$ we obtain

$$
\log \left(\Phi^{\prime}(y)\right)+\frac{y}{4}=\frac{1}{4}+\log \left(\Phi^{\prime}(1)\right)-\int_{1}^{y} \frac{S(z)}{2 z} d z \rightarrow \text { const as } y \rightarrow \infty .
$$

Hence $\lim _{y \rightarrow \infty} \Phi^{\prime}(y) e^{y / 4}=\ell \in(0, \infty)$, and (16) follows immediately.

It is clear from (11)-(12) and (15) that the uniqueness problem for $\Phi$ and that for $S$ with the prescribed value of

$$
\frac{\widehat{M}}{2 \pi}=\Phi(\infty)=\frac{\tau}{4} \int_{0}^{\infty} S(z) d z
$$

are equivalent. We recall from [3, Lemma 4.1] the idea of proving such a uniqueness property. First, the uniqueness of solutions of the Cauchy problem associated with (15) (or (11)) is established. Next, if $\Phi_{1}^{\prime}(0)<\Phi_{2}^{\prime}(0)$, then $\Phi_{1}(y)<\Phi_{2}(y)$ for each $y>0$. Finally, $\lim _{y \rightarrow \infty} \Phi_{1}(y)<\lim _{y \rightarrow \infty} \Phi_{2}(y)$ should be proved. Unfortunately, we are not able to show the last two properties.

Despite some singularities of the coefficients, the uniqueness for the Cauchy problem (15) with a given $S^{\prime}(0) \in(0, \infty)$ holds; we recall here $[3,(50)]$ where this was the first step in the proof in the chain (50)-(55) [3].

Lemma 2.2. Let $S_{1}, S_{2}$ be two solutions of (15), $S_{1}(0)=S_{2}(0)$. If $S_{1}^{\prime}(0)=S_{2}^{\prime}(0) \in$ $(0, \infty)$, then $S_{1}(y)=S_{2}(y)$ for each $y>0$.

The following result, see $[3,(53)]$, is the key point in proving Lemma 2.2 .

If $n \in \mathcal{C}^{1}\left(\left[0, Y_{0}\right)\right)$ satisfies the linear second order differential equation

$$
n^{\prime \prime}(y)+a(y) n^{\prime}(y)+b(y) n(y)=0, \quad y \in\left(0, Y_{0}\right),
$$

with $a(y) \geq 0, b \in \mathcal{C}^{1}\left(\left(0, Y_{0}\right)\right)$ such that $b(y) \geq 0, b^{\prime}(y) \leq 0, b(y)=\mathcal{O}\left(y^{-2}\right)$ as $y \rightarrow 0$, and $n(0)=n^{\prime}(0)=0$, then

$$
n(y)=0 \quad \text { for } \quad y \in\left(0, Y_{0}\right) .
$$

Indeed, we multiply (21) by $n^{\prime}$ and integrate over $(0, Y), 0<Y<Y_{0}$, to obtain

$$
\frac{1}{2}\left(n^{\prime}\right)^{2}(Y)+\int_{0}^{Y} a(z)\left(n^{\prime}(z)\right)^{2} d z+\frac{1}{2} b(Y) n^{2}(Y)-\frac{1}{2} \int_{0}^{Y} b^{\prime}(z) n^{2}(z) d z=0,
$$

the boundary terms at $y=0$ vanishing thanks to the assumptions on $b$ and $n$. Since both $a$ and $-b^{\prime}$ are nonnegative, we conclude that $n^{\prime}(Y)=0$ for each $Y \in\left(0, Y_{0}\right)$, whence (22). 
Now, putting $n=S_{2}-S_{1}$, it follows from (15) that $n$ satisfies $n(0)=n^{\prime}(0)=0$ and $n^{\prime \prime}(y)+a(y) n^{\prime}(y)+b(y) n(y)=0$ with

$$
a(y)=\frac{\tau+1}{4}+\frac{\left(S_{2}+S_{1}\right)(y)}{4 y} \text { and } b(y)=\frac{\tau}{16}+\frac{\left(S_{2}+S_{1}\right)^{\prime}(y)}{4 y}+\frac{\tau}{4} \frac{\left(S_{2}+S_{1}\right)(y)}{2 y} .
$$

Owing to the regularity properties of $S_{1}$ and $S_{2}$ already established, $a$ and $b$ fulfill the requirements needed to apply the previous result (in particular, $b$ is a sum of products of two strictly decreasing functions in the neighborhood of $y=0$ since $S_{1}, S_{2}$ are concave there), whence the conclusion.

LEMma 2.3. For any $\tau>0$ and each solution $(\Phi, S)$ of (11) we have $S(y)<4$.

Proof. The case $\tau \in(0,1]$ has already been considered in [7, Prop. 6.1]. The idea was to use the second equation in (11) represented as in (14). Then the first equation in (11) was multiplied by $\exp (\tau y / 4)$ to get

$$
\left(e^{\tau y / 4} \Phi^{\prime}(y)\right)^{\prime}+\frac{1-\tau}{4} e^{\tau y / 4} \Phi^{\prime}(y)+\frac{1}{2 y} e^{\tau y / 4} \Phi^{\prime}(y)\left(e^{-\tau y / 4} \int_{0}^{y} e^{\tau z / 4} \Phi^{\prime}(z) d z\right)=0,
$$

and dropping the second term on the left hand side (which is positive for $\tau \leq 1$ ) we obtain

$$
W^{\prime \prime}(y)+\frac{1}{2 y} W^{\prime}(y) e^{-\tau y / 4} W(y) \leq 0
$$

for the auxiliary function $W(y)=e^{\tau y / 4} S(y)$, which yields

$$
S(y) \leq \frac{\tau y}{e^{\tau y / 4}-1}<4, \quad 0<\tau \leq 1
$$

cf. $[7,(6.8)]$.

If $\tau>1$, we take into account the second term in (23), and rewrite the equation for $W$

$$
W^{\prime \prime}(y)+\frac{1-\tau}{4} W^{\prime}(y)+\frac{1}{2 y} W^{\prime}(y) e^{-\tau y / 4} W(y)=0
$$

as

$$
y W^{\prime \prime}(y)+\frac{1-\tau}{4} y W^{\prime}(y)+\frac{1}{4}\left(W^{2}(y)\right)^{\prime} e^{-\tau y / 4}=0 .
$$

Then we integrate the equation and divide by $W^{2}$ to obtain $\frac{y W^{\prime}-W}{W^{2}}+\frac{1-\tau}{4} \frac{y}{W}-\frac{1}{W^{2}} \frac{1-\tau}{4} \int_{0}^{y} W(z) d z+\frac{1}{4} e^{-\tau y / 4}+\frac{1}{W^{2}} \frac{\tau}{16} \int_{0}^{y} W^{2}(z) e^{-\tau z / 4} d z=0$.

Dropping the third and the fifth (nonnegative!) terms on the left hand side we arrive at

$$
\frac{1}{4} e^{-\tau y / 4} \leq\left(\frac{y}{W(y)}\right)^{\prime}+\frac{\tau-1}{4}\left(\frac{y}{W(y)}\right)
$$

Finally, considering the quantity $R(y)=\exp \left(\frac{\tau-1}{4} y\right) \frac{y}{W(y)}$, we obtain $\frac{1}{4} e^{-y / 4} \leq R^{\prime}(y)$, and thus

$$
W(y) \leq \frac{y e^{\tau y / 4}}{\left(1+1 / \Phi^{\prime}(0)\right) e^{y / 4}-1}<\frac{y e^{\tau y / 4}}{e^{y / 4}-1}
$$

from which

$$
S(y) \leq \frac{y}{e^{y / 4}-1}<4, \quad \tau>1,
$$

readily follows. 
Lemma 2.4. If $\tau \in[0,1 / 2]$, then $\frac{\widehat{M}}{2 \pi}<4$.

Proof. Consider the function $L(y)=(\Phi(y)-2)^{2}+4 y \Phi^{\prime}(y)$ reminiscent of that in the proof in [1, Prop. 3 (i)]. $L$ satisfies the relation

$$
L^{\prime}(y)=2 \Phi^{\prime}(y)\left(\Phi(y)-S(y)-\frac{y}{2}\right)
$$

so that

$$
\begin{aligned}
\left(\frac{\widehat{M}}{2 \pi}\right)^{2}-4 \frac{\widehat{M}}{2 \pi} & =L(\infty)-L(0)=\int_{0}^{\infty}\left(\Phi(y)-\frac{\widehat{M}}{2 \pi}\right)^{\prime}(2 \Phi(y)-2 S(y)-y) d y \\
& =-\int_{0}^{\infty}\left(\frac{\widehat{M}}{2 \pi}-\Phi(y)\right)\left(1-\frac{\tau}{2} S(y)\right) d y
\end{aligned}
$$

Now, for $\tau \leq 1 / 2$ the integrand in the last term is strictly positive since $\Phi(y)<\frac{\widehat{M}}{2 \pi}$ and $S(y)<4$ by Lemma 2.3 , so that $\Phi(\infty)=\frac{\widehat{M}}{2 \pi}<4$ follows.

LEMMA 2.5. If $\tau \in(1 / 2,1]$ then $\frac{\widehat{M}}{2 \pi}<\frac{2}{3} \pi^{2}$. If $\tau>1$ then $\frac{\widehat{M}}{2 \pi}<\min \left(\tau \frac{2}{3} \pi^{2}, 4(\tau+1)\right)$.

Proof. Since (20) holds, (24) yields the relation

$$
\frac{\widehat{M}}{2 \pi}=\Phi(\infty)<4 \int_{0}^{\infty} \frac{z}{e^{z}-1} d z=\frac{2}{3} \pi^{2}
$$

for $1 / 2 \leq \tau \leq 1$.

On the other hand, (25) leads to

$$
\frac{\widehat{M}}{2 \pi}=\Phi(\infty)<\frac{\tau}{4} \int_{0}^{\infty} \frac{y}{e^{y / 4}-1} d y=\tau \frac{2}{3} \pi^{2}
$$

for $\tau>1$, which improves the estimate $\frac{\widehat{M}}{2 \pi}<\tau^{2} \frac{2}{3} \pi^{2}$ in [7, Prop. 6.1].

Rewrite the first equation in (11) in the form

$$
\left(4 y \Phi^{\prime}(y)\right)^{\prime}-4 \Phi^{\prime}(y)+y \Phi^{\prime}(y)+2 \Phi^{\prime}(y) S(y)=0 .
$$

Integrating the last term in $(26)$ over $(0, \infty)$ we obtain using $(11)$

$$
\begin{aligned}
\int_{0}^{\infty} \Phi^{\prime} S & =\frac{4}{\tau} \int_{0}^{\infty} \Phi^{\prime}\left(\Phi^{\prime}-S^{\prime}\right)=-\frac{4}{\tau} \int_{0}^{\infty} \Phi^{\prime \prime}(\Phi-S) \\
& =\frac{4}{\tau} \int_{0}^{\infty}\left(\frac{1}{4} \Phi^{\prime}+\frac{1}{2 y} \Phi^{\prime} S\right)(\Phi-S) \\
& =\left.\frac{1}{2 \tau} \Phi^{2}\right|_{0} ^{\infty}-\frac{1}{\tau} \int_{0}^{\infty} \Phi^{\prime} S+\frac{2}{\tau} \int_{0}^{\infty} \frac{1}{y} \Phi^{\prime} S(\Phi-S) .
\end{aligned}
$$

Since $\Phi^{\prime}(y)>0, \Phi(y)-S(y)=\frac{\tau}{4} \int_{0}^{y} S(z) d z$, we arrive at

$$
2 \int_{0}^{\infty} \Phi^{\prime} S \geq\left(1+\frac{1}{\tau}\right)^{-1} \frac{1}{\tau}\left(\frac{\widehat{M}}{2 \pi}\right)^{2}=(\tau+1)^{-1}\left(\frac{\widehat{M}}{2 \pi}\right)^{2}
$$

(this was done integrating $(26)$ over $(0, \infty)$ and using $(19))$. Hence we have $-4\left(\frac{\widehat{M}}{2 \pi}\right)+$ $(\tau+1)^{-1}\left(\frac{\widehat{M}}{2 \pi}\right)^{2} \leq 0$, that is: $\frac{\widehat{M}}{2 \pi} \leq 4(\tau+1)$ which is a better bound than $\frac{\widehat{M}}{2 \pi}<\tau^{2} \frac{2}{3} \pi^{2}$ for $\tau>4\left(\frac{2}{3} \pi^{2}-4\right)^{-1} \approx 1.5505 \ldots$ 
Using essentially the same type of argument as in [3, Proposition 4.2] we may obtain the following result for radially symmetric solutions of the system (1)-(2):

Proposition 2.6. Let $(\varphi, \psi)$ be a solution to the problem (8). Then there exists a selfsimilar solution $\varphi^{\infty}(s, t)=\Phi^{\infty}(s / t)$ with $\Phi^{\infty}$ satisfying (11) such that

$$
\lim _{t \rightarrow \infty}\left\|\varphi(t)-\varphi^{\infty}(t)\right\|_{L^{\infty}}=0 .
$$

In fact, $\varphi^{\infty}(s, t)=\sup _{\lambda>0} \varphi(\lambda s, \lambda t)$.

Note that if we had the uniqueness of self-similar solutions of (11), the conclusion might be strengthened to:

For the self-similar solution $\Phi_{\widehat{M}}$ corresponding to the mass $\widehat{M}$,

$$
\lim _{t \rightarrow \infty}\left\|\varphi(t)-\Phi_{\widehat{M}}(t)\right\|_{L^{\infty}}=0
$$

Acknowledgements. I thank Yūki Naito and Tadeusz Nadzieja for pertinent remarks. The preparation of this note was partially supported by the KBN (MNI, MEiN) grant 2/P03A/002/24 and by the European Commission Marie Curie Host Fellowship for the Transfer of Knowledge "Harmonic Analysis, Nonlinear Analysis and Probability" MTKDCT-2004-013389.

\section{References}

[1] P. Biler, Growth and accretion of mass in an astrophysical model, Appl. Math. (Warsaw) 23 (1995), 179-189.

[2] P. Biler, Local and global solvability of some parabolic systems modeling chemotaxis, Adv. Math. Sci. Appl. 8 (1998), 715-743.

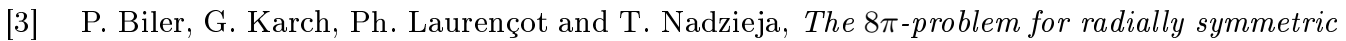
solutions of a chemotaxis model in the plane, Math. Methods Appl. Sciences 29 (2006), $1563-1583$.

[4] A. Blanchet, J. Dolbeault and B. Perthame, Two dimensional Keller-Segel model: Optimal critical mass and qualitative properties of the solutions, Electron. J. Diff. Eqns. 44 (2006), $1-33$.

[5] Y. Naito, Non-uniqueness of solutions to the Cauchy problem for semilinear heat equations with singular initial data, Math. Ann. 329 (2004), 161-196.

[6] Y. Naito, Asymptotically self-similar solutions for the parabolic system modelling chemotaxis, this volume, 149-160.

[7] Y. Naito, T. Suzuki and K. Yoshida, Self-similar solutions to a parabolic system modeling chemotaxis, J. Differential Equations 184 (2002), 386-421. 